\title{
Informing the Design of Proxemic Interactions
}

\author{
Nicolai Marquardt and Saul Greenberg \\ Department of Computer Science, University of Calgary \\ 2500 University Dr NW, Calgary, AB, T2N 1N4, CANADA \\ [nicolai.marquardt, saul.greenberg] @ucalgary.ca
}

\begin{abstract}
Proxemic Interactions envision interactive computer systems that exploit peoples' and devices' spatial relationships (proxemics) to provide more natural and seamless interactions with ubicomp technology. It builds upon fundamental proxemic theories about people's understanding and use of the personal space around them. In this paper, we focus on how nuances of the proxemic theories and concepts of Proxemic Interaction can be applied to address six key challenges of ubicomp interaction design, where we consider how we can leverage information on fine-grained proxemic relationships. We also discuss how previous proxemic-aware systems addressed these challenges.
\end{abstract}

ACM Classification: H.5.2 [Information interfaces and representation $(\mathrm{HCI})]$ : User Interfaces: Input devices and strategies; theory and methods.

General terms: Design, Human Factors

Keywords: Proxemics, interaction, ubiquitous computing

\section{INTRODUCTION AND BACKGROUND}

Social scientists and others in related fields describe Proxemics as people's cultural perception and use of personal space to mediate their social interactions with others in everyday situations [1]. While proxemics emphasises distances between people, other attributes are also relevant, e.g., orientation and body language. Yet, despite people's understanding of proxemics, only a handful of interactive systems within Pervasive and Ubiquitous Computing (ubicomp) [2] have applied proxemic relationships to interaction design in a holistic way e.g., $[3,4]$. This is surprising, since one promise of ubicomp is to situate technology in people's environments, where it leverages, exploits, and becomes integrated into everyday practice [5].

In our own recent work, we proposed the idea of proxemic interactions [6,7]. We described how devices could have

Cite as:

Marquardt, N., Greenberg, S. (2011)

Informing the Design of Proxemic Interactions.

Research Report 2011-1006-18, Department of Computer Science, University of Calgary, Calgary, AB, Canada T2N 1N4, July. fine-grained knowledge of nearby people and other devices, and - as others have also done - we illustrated various examples of how that knowledge could be exploited to design interaction techniques.

In this article, we take a step back. We focus on proxemic theory and show its potential to address five key design challenges of ubicomp interaction [8]: revealing interaction possibilities, directing actions, establishing connections, providing feedback, preventing and correcting mistakes, and managing privacy and security. We then operationalize proxemics as knowledge that can be sensed or captured by devices via five essential dimensions - distance, orientation, movement, identity, and location - and discuss the nuances of their use. We then relate both the theory and dimensions to the design challenges, and situate a sampling of prior systems within that setting [e.g., 3,4,6].

\section{UBICOMP AND EMBODIED INTERACTION}

Almost twenty years ago, Mark Weiser proposed Ubiquitous Computing (ubicomp) as the next era for interacting with computers [2]. In this proposed future, he foresaw that network connected digital technologies would be available in our everyday environments, in a variety of form factors and sizes that would suit the task at hand. Given today's availability and use of such devices smartphones, tablet computers, net-aware digital cameras, photo-frames, interactive whiteboards, digital tabletops and so on - it may seem that his vision has been realized. But Weiser's vision went beyond devices. Importantly, he predicted the move of computing technology into people's everyday surrounding, embedded in all kind of everyday objects and spaces, where it would be seamlessly accessible:

"The most profound technologies are those that disappear. They weave themselves into the fabric of everyday life, until they are indistinguishable from it" [2].

To partially realize seamlessness, Weiser and Brown also proposed technology that "engages both the center and periphery of our attention" [9]. It is these parts of Weiser's vision - the seamless interaction, the disappearing technology, the seamless transitions between foreground engaging activity and background peripheral perception that is still missing from people's everyday experience with ubicomp technology. 
Later, Dourish expanded upon the concept of situating technology in people's everyday environment as embodied interaction [5]. Dourish brought together the core ideas of phenomenology theory, social computing, and tangible user interfaces, where he emphasized the importance of designing technology that exploits human skills and experiences that takes place in their world [5]. Extending the ubicomp vision, the goal of embodied interaction is to build technology that is seamlessly integrated into people's everyday practices. People should not act on technology but instead through the technology, to perform their task at hand [5]. Context-aware computing relates to this, where: some kind of context-aware sensing method [10] provides devices with knowledge about the situation around them; could infer where they are in terms of social action; and could act accordingly.

\section{CHALLENGES IN DESIGNING UBICOMP SYSTEMS}

In this section, we introduce six core challenges in the design of embodied and seamless interaction within ubicomp, with an emphasis on their relevance to proxemic interactions. Our challenges are inspired by Bellotti's [8] important design considerations for sensing systems, augmented by issues raised in other analytical and reflective ubicomp discussions $[3,11,12,13,14]$.

Challenge 1. Revealing interaction possibilities [12], [13]: Norman [15] appropriated Gibson's [16] notion of affordances to describe how an object's visuals can 'suggest' how it might be used. Traditional GUIs exploited affordance to design interface elements that suggested their use and possible actions; they worked, because they could assume that they were in the foreground of a user's attention, i.e., the person was watching the screen. Yet this cannot be directly applied to ubicomp, as ubicomp assumes that technology can be integrated into the everyday environment in a way that it 'disappears', or is present in the just-perceptible periphery of our attention, and that it is able to fluently move into the center of our attention as needed $[2,12,17]$. This introduces the challenge: how can technology be designed to reveal the interaction possibilities appropriate when it is not only in the background of a person's attention, but during the transition of it moving into the foreground?

Challenge 2. Directing actions [3,8,14]: Input to a single traditional device is straightforward, as it usually comes through a dedicated input device (e.g., a mouse, keyboard, touch surface). Yet ubicomp can be different. Input may be detached from a particular device. Possible actions can be performed through speech, gestures, eye gaze and other alternative options. One problem is that the device has to somehow discern whether that action is actually a directive to the system, or whether it should be ignored [8] because it is just part of a person's everyday actions (e.g., a voice command vs. social talk; a command gesture $v s$. a gesture or movement made in the course of doing other things). The problem of directing the actions to a particular device is

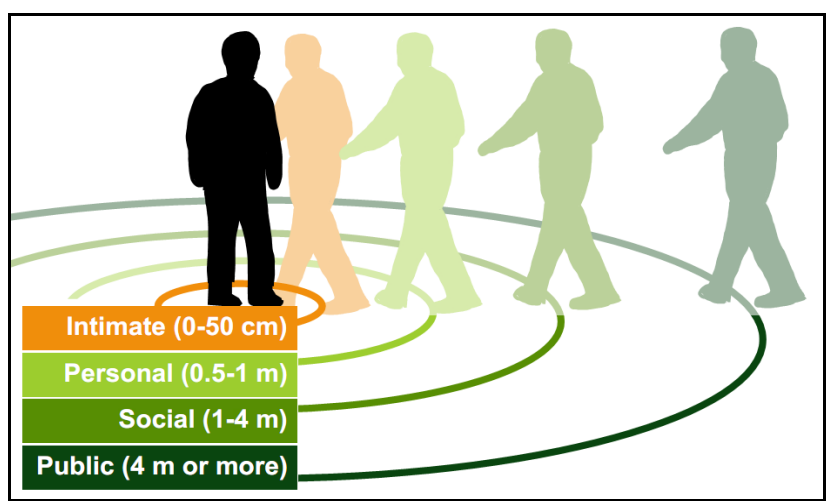

Figure 1. Hall's proxemic zones.

even more problematic when there are large quantities of devices present in the local ecology, for the system has to discern which device (or set of devices) should respond to a person's directed action.

Challenge 3. Establishing connections [12,18]: Device connectivity is a significant challenge in ubicomp. Technical issues aside, ubicomp's ad-hoc nature means that people need to somehow control (albeit seamlessly) how one device connects to another device in a way that reflects their interaction needs while still safeguarding privacy and security (for example to transfer digital content from a personal smartphone to a large public screen). This challenge is compounded by the potential and perhaps unpredictable interplay between a large numbers of digital devices. Some may be personal (a smart phone), others may belong to the inhabitants of a space (a home's picture frame), and others may be public (e.g., a public wall display). Their form factor also affects their mobility, which in turn can suggest different factors affecting how they should establish connections.

Challenge 4. Providing feedback, [3,8,11,14]. Appropriate feedback is a mainstay of traditional GUI interaction design. Yet as ubicomp interfaces move away from the traditional desktop computer setting, it becomes even more important to provide feedback about the current status of the application, its interpretation of user input, or the occurrence of errors. To complicate matters, ubicomp systems have to consider that people's attention in regards to the ubicomp technology might switch between foreground and background.

Challenge 5. Avoiding and correcting mistakes [3,8]: When mistakes or errors happen, the system should provide options for a person to correct these mistakes. As many ubicomp systems use some kind of sensing technology to monitor people's actions, such errors and misinterpretation of sensor data are even more likely to occur in ubicomp settings than with traditional computers.

Challenge 6. Managing privacy and security [19]: A large issue in ubicomp is that as the number of potential interactions with technology increase, so too do the risks to 
privacy and the need for greater security. The question is how can the system protect privacy sensitive information and handle the access to information, while at the same time not get in the way of all the positive offerings of ubicomp mentioned in Challenges 1-5?

\section{PROXEMIC THEORIES}

We will shortly explain techniques based on proxemic interaction that can mitigate problems inherent in these challenges. However, we will first set the scene by summarizing aspects of proxemic theories and use of personal space that we believe are relevant to ubicomp design.

\section{Distances and Discrete Zones}

Anthropologist Edward Hall introduced proxemics as a theory for studying the interpersonal spatial relationships between individuals [1]. His theory - while emphasising social and cultural differences - generally describes how people perceive, interpret, and (often unconsciously) use the micro-space around them, and how this affects their interaction and communication with other nearby people. $\mathrm{He}$ details how people perceive, interpret and use proxemics cues, especially distance, to mediate relations to other people. In particular, he correlates physical distance to social distance between people. As illustrated in Figure 1 , he categorizes this into four discrete distance zones, ranging from: intimate $(0-50 \mathrm{~cm})$, personal $(0.5-1 \mathrm{~m})$, social $(1-4 \mathrm{~m})$, and public $(>4 \mathrm{~m})$. These collective distances, which Hall calls the dynamic space, characterize a progression of interactions ranging from highly intimate, to personal, to social and then to public [1]. The exact ranges of these interpersonal distance zones depend not only on cultural factors, but also on other factors such as age, gender, or personal relationship [20].

\section{Environment: Fixed and Semi-Fixed Features}

Hall also identified two other factors that influence people's use of the micro-space around them [1]. Fixed features include the immobile properties of the space: the layout of buildings and rooms, the walls, doors, and windows. Semifixed features include the spatial layout of elements in the space that can be moved (like furniture, chairs, or tables). Hall noticed that the layout of the fixed features as well as the arrangement of elements in the semi fixed feature space influence our use and perception of personal space, where particular layouts can be sociofugal (separating people) and sociopetal (bringing people together) [21]. A simple example is how chairs in a living room can be brought together into a sociopetal small circle to encourage intimate chat.

Although others have critiqued Hall's classification of personal space as being overly simple (e.g., comments in [22]), his work has become a seminal theory of studying personal space. Since then (and summarized next), other theories added new perspectives that go beyond Hall's original distance-centric view.

\section{Orientation}

Orientation generally describes how people face towards or away from each other, and this too affects proxemic relationships. Sommer [23] studied people's preference of spatial seating arrangements and relative orientation around a table depending on the task at hand. Depending on the task, the majority of people tended to particular seating positions: face-to-seating face for competitive tasks, sideby-side for cooperative tasks, and side-by-side or corner-tocorner during conversations. Others identified patterns, where people's orientation to one another depended on the type of conversion and social status [24]. In later work, Hall included orientation as an essential variable in describing proxemic relationships [25].

\section{Compensation, Balance, and Privacy}

People constantly adjust their use of space to fit the presence of, and interactions with, others. This includes how people react to and try to overcome 'invasions' or 'violations' of their personal space. Some theories describe people's adaption to given spatial circumstances, and how they try to maintain a certain comfort level or equilibrium in these situations [26]. For example, the intimacy equilibrium model [27] assumes that when people interact they always strive to maintain an overall balance towards a desired optimal proxemic distance. To achieve this balance, people might try to adapt proxemic variables such as distance, orientation, or eye contact, which the model describes as "inverse relationship between mutual gaze, a nonverbal cue signaling intimacy, and interpersonal distance" [24]. For example, when a person stands too close to us, we might step back to maintain the equilibrium. If any of the variables cannot be changed in this particular situation (such as standing very close to others in an elevator), the change of another variable can be used to compensate (in the elevator example: changing orientation to face away while avoiding eye contact). Another predictive model formalizes equilibrium as an optimal proxemic distance, where it adds proxemics variables including identity and familiarity of the other person, and the type of interaction [28]. People also use personal space as a method to protect a certain level of privacy. Altman [29] reframes this use as a dynamic boundary regulation process that controls privacy.

\section{PROXEMIC DIMENSIONS FOR UBICOMP INTERACTION}

The proxemic theories above describe many different factors and variables that people use to perceive and adjust their spatial relationships with others. We recognize that these theories describe people's relations to people, and not to devices. Even so, our belief is that we can use these theories as a first-order approximation to apply proxemics to ubicomp design. As part of this approximation, we offer five device-oriented proxemic dimensions - inputs and states that devices can hold about proxemics relationships that we believe are most relevant to operationalizing proxemics in ubicomp interaction [6,7]. That is, they 


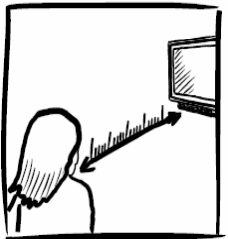

Distance

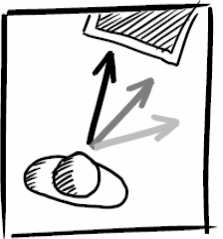

Orientation

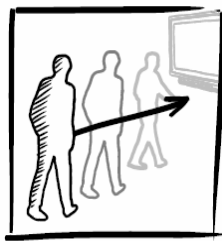

Movement

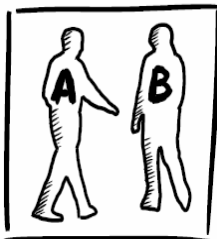

Identity

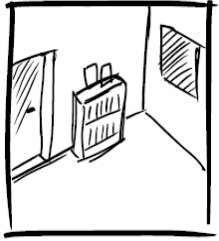

Location
Figure 2. Proxemic dimensions relevant for ubicomp interaction.

problems inherent in each challenge. Our examples are merely a starting point, where their contributions are reframed within each challenge, and where they hint at the potential of future proxemic interaction designs.

\section{Challenge 1: Revealing Interaction} Possibilities

To address this challenge, a system describe not only relationships between person-to-person, but with all entities in ubicomp ecologies: people, digital devices, non-digital objects, and the features of the surrounding environment. The list below describes each dimension, while Figure 2 illustrates each as a graphic.

- Distance: describes the measurable distance between entities in the space (people, devices, and objects). Distances can be represented in many ways. For example, they can be precise (e.g., 2.3 meters) or crude (e.g., zone 1), given as absolute positions or as relative distances between entities, and provided continuously as changes are detected, or discretely as a distance passes from one zone to another.

- Orientation: provides information about which direction an entity is facing. For people, this includes gaze, face, and body orientation, as well as the orientation of limbs like arms. For devices or objects, this might require a well-defined front (e.g., the frontfacing side of a display). Orientation can be relative between two or more entities, or absolute when relative to a fixed point in the environment. They can be described in both qualitative (e.g., "facing towards") and quantitative (e.g., the measured angle) terms.

- Movement: describes the changes of distance and orientation over time. Thus it can describe sequences of an entity moving through space, and even its velocity.

- Identity: uniquely identifies entities in the space. This can be an exact identification (e.g., Fred, Jane), or an identity that discriminates one entity from another (e.g., person 1 from person 2), or a category ("person", "phone").

- Location: in contrast to the earlier mentioned distance, the dimension location describes the qualitative aspects of the place where the interaction takes place. That is, it characterizes the location (such as home vs. office settings), describes features in the fixed (e.g., room layout) or semi-fixed (e.g., furniture positions) feature space, and provides meta-information such social practices and context of use of that space by the entities seen within it.

\section{ADDRESSING UBICOMP DESIGN CHALLENGES WITH PROXEMICS}

We now revisit each design challenge, where we speculate - with examples drawn from the literature - how knowledge of proxemics as gathered by the 5 dimensions can mitigate must offer possible actions [3] that afford seamless transitions from background to foreground interaction [17]. This concept is somewhat similar to how people approaching each other exchange greet and begin communicating through various signals (eye gaze, body language and talk), where signals and possible actions vary appropriately across this greeting phase. Similarly, ubicomp should 'greet' other entities by revealing interaction possibilities that match what is possible at the moment. Several strategies to accomplish this are described below.

Reacting to the presence and approach of people. At the most basic level, if a system can sense the presence and approach of people, it can use that information to reveal possible interactions.

Various prior systems do this, but only as a binary measure: if it detects a person it marks them as 'present', otherwise 'absent. In response to this binary measure, systems would trigger an appropriate action. In [17], smart light switches used motion detectors to infer presence and then turn lights on and off in response. In [7], a desktop computer screen used a proximity sensor to capture a person's distance from the display, and from that either activated the screen or went into a power-save mode. Both systems 'reveal' interaction possibilities implicitly: the first by illuminating the room, and the second by showing the desktop computer is on and ready to go.

Other systems detect and use presence information to explicitly reveal interaction possibilities. Consider ActiveBadges - identity tags worn by individuals - where badge (and thus a particular person's) location is tracked at a room-level within a building [30]. Its inventors exploited this presence and identity information to offer personalized computing services at that person's current location, e.g., where their desktop computer display would 'follow' them to other rooms and appear on nearby screens. Another popular example is a large screen that senses when a person enters a room, where the display not only turns on but tailors its contents to suggest its offerings [4,6]. In [6], for example, when a person crosses a threshold into a room, a splash screen appears revealing that the large display is a media player, and then offers several videos the person could select for watching. The system intentionally displays only a small number of videos using large graphics, to make it appropriate for viewing at a distance. If one person seated on a couch is already viewing a video while another person enters, the system displays its information differently, 
where it reveals what is being viewed with minimal disruption to the primary viewer. In terms of our dimensions, this media player exploits both relative distance and identity to reveal appropriate interaction possibilities compared to just a binary notion of presence, i.e., it considers the room as an ecology. It uses people's approach across a threshold (the doorway), their distance from the screen, and their presence relative to other fixed features in the room (e.g., the couch).

From awareness to interaction. In real life, people exploit proxemics cues as they greet and engage in social interaction. One may have peripheral awareness of the other while at a distance, become increasingly aware and engaged as the other turns towards and approaches them, and then begin to interact when within an appropriate proxemic region. Some public ambient displays apply a similar mechanism to engage people, where they trigger actions to attract a passer-by's attention, and progressively show more information and interaction possibilities as the person approaches and attends the display, ideally leading to foreground interaction by direct touch $[4,6,31,32]$. The idea is that the passer-by notices the public display as it implicitly reacts to their presence, where it captures their attention and interest (as discussed in Challenge 1). Their attention is realized by moving closer and facing the display; the system also detects and react to that interest $[4,31]$. In the media player [6], for example, the number of videos, their size and associated text is adjusted as the person approaches the display, where it reveals more video selections and more information about those videos. A system such as this exploits distance, orientation and movement to infer a person passing by at a larger distance, then turning towards the display, then approaching, and finally standing directly in front of it.

Spatial visualizations of ubicomp environments. In the physical world, we often know what is available simply by looking around us. Ubicomp, however, participates in both the physical and virtual, so not all offerings are readily visible. One way around this is to spatially visualize otherwise hidden offerings on a device's screen(s). Consider the problem of several people in a room, each with a mobile device, and how one knows when one device is within range of another, and what can subsequently be done with them. To solve this, Gellersen's Relate Gateways [33] provides a graphical map on each mobile device's screen showing the nearby devices that it senses. Icons on the map indicate the position of devices that can be accessed. In an alternate visualization, icons at the border of the screen represent the type and location of surrounding devices relative to that device's position. In our media player example [6], the display visualizes the spatial relationships to nearby personal devices by using a method akin to ray-casting: if a person points their device towards the large screen, a graphic appears as a ray-cast 'projection' on that screen indicating its position and orientation. As the mobile device approaches and is oriented towards the large display, increasing detail about that device, its contents and its interaction possibilities are revealed.

\section{Challenge 2: Directing Actions}

While Challenge 1 concerns how a ubicomp system can reveal interaction possibilities to a person, Challenge 2 addresses how a person can in fact direct their input actions to a particular device.

Discrete distance zones for interaction. Similar to how people tend to move closer to others when interacting (say, to begin a conversation), systems might accept user input only when the person has a certain distance relative to the device. Thus, to address a particular system, a person may have to approach and move closer to it. Some ambient display systems do this by realizing Hall's discrete proxemic zones as thresholds that adjust interaction possibilities according to which zone a person is in $[4,32]$. Hello.Wall [32] introduced the notion of distancedependent semantics, where the distance of an individual from the wall defined the kinds of interactions possible. While information on the large display can be seen from afar (Challenge 1), a person had to move closer to actually interact with it (e.g., to transfer information from a mobile device). Vogel et al. [4] extended this concept, where they defined four proxemic zones of interaction around the large display. From far to close, these ranged from ambient display, to implicit, then subtle, and finally to personal interaction with the interactive calendar application. Each of these zones allowed particular kinds of interaction with the display's contents. Similarly, Ju [3] also defined four zones around an interactive whiteboard, where she allows certain actions only when a person is standing close to it. Our media player shows yet another promising approach, where each interaction zone explicitly support different input modalities that are appropriate to the person's distance from the display [6]. When afar, people interact via pointing (ray casting) and by hand gestures direct touch when in close distance.

Considering attention and orientation. Instead of relying on only distance, the system can use other measures to infer a person's attention to it. This is the premise of attentive user interfaces (AUIs) that are designed to "support users' attentional capacities" [34]. In one class of AUIs, the system reaction depends on whether a person is directing his or her attention to the device as detecting eye gaze [34], which in turn can be considered a very fine-grained measure of orientation. Our media player also exploits orientation as a measure of attention [6]. When a person turns away from the video screen (to, say, read a magazine or talk to another person), the system pauses video playback, and resumes when they turn back towards it. Wang's proxemic-aware presenter [35] also uses orientation as an indication of attention. If the presenter is facing towards the audience and away from the large display, a standard slide deck is shown. However, when the 
presenter turns towards the display, small navigation controls and speaking notes become visible at the side of the screen closest to the presenter.

Considering location features. Ubicomp systems are often embedded in people's everyday environments, surrounded by other physical objects and social meanings that comprise the ecology of that place. Inspired by research in contextand location-awareness [10], our next concept emphasizes the importance of interpreting the physical setting where an interaction takes place [5]. In particular, people's relationships to fixed and semi fixed features (as defined by Hall [1]) can be indicators for directing actions to a particular ubicomp system. In our media player [6], the ubicomp system not only monitors a person's proxemic relationship towards a device, but also to that person's distance to other fixed and semi-fixed features in the ecology. If a person selects a video and then sits on the couch, that is interpreted as an indicator that she is ready to watch the currently selected video and thus video playback begin. However, if the person instead moves to the doorway, that is interpreted as an indicator that she is no longer interested, and the system shuts down. In both cases, the distance from the person to the screen is the same, but her location in the room's ecology is different.

Motion trajectories. Going straight towards another person - or instead quickly passing by - are also proxemic cues that we implicitly interpret in everyday interactions with others. Similarly, ubicomp systems can interpret people's and device's motions for directing actions. For example, Vogel's [4] ambient display ignores people quickly passing by, but reacts to (and gathers input) from people walking straight towards it. Motion cues can be quite fine-grained, where it can exploit distance, orientation and velocity as well as how each changes over time.

Adapt to number of nearby devices. A system's interpretation of a person's actions can also depend on the number of other nearby devices that it can sense. To illustrate, consider Swindells [36] gesturePen that allows device to device interaction from a distance when one device is pointed to the other device. We foresee extending this technique to a large number of devices by applying further distance-or identity-based filtering technique to limit the number of possible pointing targets. When pointing towards a large number of possible targets, the system could require the person to move closer to do a precise selection of the target device. In contrast, if only a single device is in that area, the system will recognize the target device and will not require the person to move closer.

\section{Challenge 3: Establishing Connections Between Devices}

As suggested by our last example, people need to somehow control how one device connects to another device within a potentially large ecology of devices in a way that seamlessly supports their interaction needs while still safeguarding privacy and maintaining security. We do this naturally - the way we greet and move closer to one another via proxemics is essentially a negotiation to establish connections for communication.

Connection as a consequence of close proximity. We can exploit distance, identity and even orientation to determine proxemic relationships between devices, and then establish connections between only those that are in close proximity. Existing systems now do this, although most do so as a binary function (e.g., close = connected). Rekimoto's combination of near-field RFID communication and wireless networks allows inter-device communication only when two mobile devices are in close proximity [37]. Alternately, physically bumping two devices together can activate a connection: the accelerometer signal produced by bumping identifies the devices [38], and bumping can only occur as a consequence of direct touch. Another strategy exploits people's proximity to on another, where they communicate to synchronize an act that establishes the connection. One example is both simultaneous shaking their handheld device [39]. Similarly, a stitching gesture can be used, where one person start a gesture on one device, which is then continued on the other; this can only be done if the devices are nearby [40].

While the above systems are binary in nature, progressive connection processes are also possible. Kray's group coordination negotiation [41] introduced spatial regions around mobile phones to establish and break device connections or initiate data transfer. As a device moves across three discrete regions, a preview of a media transfer is first display, where transfer begins only after moving into a closer region. Our proxemic media player is somewhat similar, but it uses a continuous rather than discrete progression over distance [6]. When a person holds a handheld media player in her hand, a subtle notification on the large screen indicates the connection possibility. As he moves closer to the screen, he sees the two devices connect, where the large display progressively reveals more information about the handheld's video content as icons. As the two devices move within touch distance, a touch interface appears that allows the person to transfer digital media either through pick and drop or by touching the handheld to one of the icons revealed on the large display.

\section{Challenge 4: Providing Feedback}

Next, we discuss how to leverage proxemics for providing continuous feedback about a system's status or any errors that occur.

Adjusting feedback output. Due to the embedded nature of many ubicomp systems, there is often no graphical display for showing feedback to the user. Instead, output can be via visual lights, audible sounds, speech, or physically moving objects (like in many tangible user interfaces). Assuming a system knows the physical orientation and distance of a person, it can adjust the provided output to the person that it is addressing. The Listen Reader [42], for example, adjusts the volume of the audio output depending on a 
person's proximity to a digitally augmented book. Similarly, in our media player [6] a person sees large preview thumbnails of available videos when at a distance. The screen continuously shows more content as the person moves closer (and thus, can read more information).

Selecting appropriate feedback modality. Furthermore a system can select the most appropriate output modality to a person (e.g., visual vs. audible) based on their proxemic relationship. For example, when the person is facing away from a large screen, the system might use an audible signal as a notification. When the person is standing closer to the system facing the screen, visual output may be used instead.

Proxemic-dependent reveal of feedback. Details presented to a person can vary depending on the distance and/or orientation of the person relative to the system. Lean and Zoom, for example, introduced a distance-dependent semantic zoom technique [43], where more details of the displayed object are revealed when the person moves her head closer to the screen. This idea of semantic zoom can be applied to ubicomp environments. He [44, chapter 3] implemented an augmented reality energy viewer for the home, where feedback of energy use was adjusted based upon the viewer's proximity to rooms or appliances within a room (distance and orientation are detected through fiduciary tags). When holding the viewer outside a room's doorway, the energy use of that room as a whole is displayed. When the person moves into the room, the energy use of each appliance is seen as a coloured glow around it; as he moves closer to a particular appliance, details of that usage appear first as a text overlay and then as a graph.

\section{Challenge 5: Preventing and Correcting Mistakes}

Our next design challenge addresses the question of how a person can correct errors, such as those that result from the system misinterpretation a person's action, or by the person performing an unintended action.

Inverting action. One technique allowing a person to correct a mistake (and thus undo a system's action) is by performing the inverse/opposite action. The system implicitly responds by reverting to the prior state. For example, in Vogel's ambient display setting [4], when a person moves closer to the screen, personal calendar information is revealed. If the person didn't want this information made public, he just steps back (and thus performs the opposite action): the personal information disappears immediately. Other proxemic dimensions can be exploited as well. For example, an action triggered by the person facing a screen can be stopped (or reverted) simply by turning away.

Explicit action to undo. $\mathrm{Ju}$ [3] presents an opposing explicit strategy to undo actions. Her application runs on the interactive whiteboard, where it implicitly responds to people's actions. This can easily result in an unwanted action (for example: automatically moving a cluster of ink strokes to the side of the display to free up space). To correct this, the person moves closer to the screen (instead of stepping back, like in Vogel's system) and grabs the cluster of ink strokes to keep it from moving.

Of course, both the above techniques can be combined to override the system. In fact, Vogel used both in his system: a person can either use a set of simple hand gestures to trigger or stop certain system functions, or just step back from the screen to have the same effect.

Proxemic safeguards. As a safeguard mechanism, actions with a high impact (e.g., deleting information, or resetting the system) could be restricted to occur only when a person is in very close proximity to a device. For example, while a person can manipulate information on an interactive whiteboard from a large distance by using remote gestures, she would have to move directly in front of the screen to delete data by (say) direct touch. Alternatively, such actions with high impact could even require a certain proxemic relationship in multiple dimensions. For example, the delete action could require a person to stand in close proximity to the screen and being oriented towards it and look at the screen simultaneously. The action could also be tentative and undoable as the person remains close by, but committed as they move away.

\section{Challenge 6: Managing Privacy and Security}

Next, we review techniques that apply proxemics for managing privacy and security in ubicomp systems [19].

Proximity-dependent authentication. Access to ubicomp systems can be granted depending on the sensed proximity of people, devices, or other objects. Bardram [45] discussed proximity-based user authentication allowing access to computers when approached by a person. The system is implemented through authentication tokens (e.g., pens) that wirelessly authorize the access of a person once in close proximity to the computer (i.e., the person stands in front of it). The tangible security [46] approach uses the measured proximity between pairs of tokens to authenticate access. For example, a person obtains access to a cell phone only as long as the physical security token he carries remains in close proximity. If the phone is lost, strangers cannot access its contents as they do not have the security token. Mayrhofer took this concept further [47], where his system leverages the shared knowledge (between the person and device) about spatial references to other devices in close proximity to authorize access. Furthermore, Rekimoto [37] combines near-field sensing techniques (such as RFID or Infrared) with wireless network communication to seamlessly establish device to device connections. Nearfield communication initiates the wireless communication channel. That is, a person must not only bring his device close to the other device, but also make sure they are in line of sight before the connection is established.

Distance-dependent information disclosure. Another strategy uses distance between entities to determine the 
amount of information that is shared between them. This approach suggests that "distance implies distrust" [48], and vice versa: closer proximity implies trust. For example, Marquardt's distance-dependent disclosure RFID tags [49] vary information transmitted between the tag and the reader depending on the distance between them. The closer the tag is to the reader, the more information is revealed. Similarly, Vogel's [4] public calendar reveals a person's personal calendar information only when the person is moving very close to the display. The information disappears immediately once the person steps back away from the display.

Proxemic-aware privacy mechanisms. While these approaches consider distance as a factor affecting access, the techniques could be further refined by considering other proxemic dimensions such as orientation, identity, or location. A person's body, face, or gaze orientation can affect the amount of information shared. For example, privacy-sensitive information shown on the display of a proxemic-aware mobile device could be visible as long as the person is looking at the screen, but hidden once looking away. Alternatively, the information might disappear once the system notices another person looking at the display. By considering the identity dimension, a system would be able to use relaxed privacy and security settings when a person is alone, but switch to more restrictive privacy and security settings when it detects any other people or devices around them (e.g., in a crowded setting). By considering location, a mobile ubicomp device could adjust its security setting depending on the type of environment; using higher level settings in an open office (where strangers may come by and try to access the device), but lower security level when at home (which is usually a much more trusted setting).

Considering people's expectations of personal space. Altman's [29] theory considers personal space as a protection mechanism for maintaining a certain level of privacy. This could be leveraged to design systems that respect people's expectations of personal space. That is, the ubicomp system can influence the simultaneous interaction of multiple people in a way that maintains such levels of privacy for everyone involved. To illustrate, let us revisit Vogel's public ambient display [4]. When people move closer to the display, they get more details about their own personal calendar visible on the screen. Thus, people stand next to each other viewing their personal calendars. When considering Altman's theory of balancing privacy through proxemics, the system could be designed to separate the large screen interaction areas of the two people. For instance, the areas for viewing personal calendars could be displayed where it depends upon a minimum distance between those people.

\section{CONCLUSION}

This paper discussed the application of proxemics in ubicomp interaction design. The intention was to inform ubicomp designers implementing proxemic-aware devices about important proxemic dimensions to consider for the design and review methods of how those can be applied to challenges in ubicomp interaction. By focusing in particular on how the knowledge in the five proxemic dimensions (distance, orientation, movement, identity, and location) can be applied to ubicomp interaction, we hope to open up a new perspective onto how proxemics can be considered when designing new ubicomp systems that react seamlessly and appropriately to people's expectations.

We concentrated on a few example systems and techniques to illustrate how our challenges can be addressed. These were chosen to inspire design thinking. They are not meant to be a complete review, nor as a catalog of solutions. We also recognize that a single technique can serve different purposes across these challenges. For example, the idea of progressive reveal of information as a person approaches a display reveals interaction possibilities (Challenge 1), affords actions being directed to it (Challenge 2), is used to establish a connection (Challenge 3), provides feedback that it is responding to the person (Challenge 4), can be used to prevent and correct mistakes by inverting actions (Challenge 5), and helps people manage privacy and security simply by moving to adjust what information is visible (Challenge 6). We believe this to be one of the strengths of proxemics: if techniques are developed with social expectations of proxemics in mind, they can likely be applied as a universal way to mediate many challenges in ubicomp.

\section{REFERENCES}

[1] E. T. Hall, The Hidden Dimension, 1st ed. Garden City, N.Y: Doubleday, 1966.

[2] M. Weiser, "The Computer for the 21st Century," Scientific American, vol. 265, p. 94, Sep. 1991.

[3] W. Ju, B. A. Lee, and S. R. Klemmer, "Range: exploring implicit interaction through electronic whiteboard design," in Proceedings of CSCW' '08, ACM, 2008, pp. 17-26.

[4] D. Vogel and R. Balakrishnan, "Interactive public ambient displays: transitioning from implicit to explicit, public to personal, interaction with multiple users," in Proceedings of UIST '04, ACM, 2004, pp. 137-146.

[5] P. Dourish, Where the Action Is: The Foundations of Embodied Interaction. The MIT Press, 2001.

[6] T. Ballendat, N. Marquardt, and S. Greenberg, "Proxemic Interaction: Designing for a Proximity and Orientation-Aware Environment," in Proceedings of ITS '10, ACM, 2010.

[7] S. Greenberg, N. Marquardt, T. Ballendat, R. Diaz-Marino, and M. Wang, "Proxemic interactions: the new ubicomp?," interactions, vol. 18, pp. 42-50, ACM, Jan-2011.

[8] V. Bellotti, M. Back, W. K. Edwards, R. E. Grinter, A. Henderson, and C. Lopes, "Making sense of sensing systems: five questions for designers and researchers," in Proceedings of CHI '02, ACM, 2002, pp. 415-422.

[9] M. Weiser and J. S. Brown, "Designing Calm Technology," PowerGrid Journal, vol. 1, p. 1, 1996.

[10] B. Schilit, N. Adams, and R. Want, "Context-Aware Computing Applications," in IEEE Workshop on Mobile Computing Systems and Applications, Los Alamitos, CA, USA, 1994, vol. 0, pp. 8590.

[11] G. D. Abowd, E. D. Mynatt, and T. Rodden, "The human experience [of ubiquitous computing]," IEEE Pervasive Computing, vol. 1, no. 1, pp. 48-57, 2002. 
[12] J. Bardram and A. Friday, "Ubiquitous Computing Systems," in Ubiquitous Computing Fundamentals, J. Krumm, Ed. Boca Raton, Florida, USA: CRC Press, 2010, pp. 37-94.

[13] J. R. Cooperstock, S. S. Fels, W. Buxton, and K. C. Smith, "Reactive Environments: Throwing Away Your Keyboard and Mouse," Communications of the ACM, vol. 40, pp. 65-73, 1997.

[14] A. K. Dey, "Context-Aware Computing," in Ubiquitous Computing Fundamentals, Edited by J. Krumm., J. Krumm, Ed. Boca Raton, Florida, USA: CRC Press, 2010, pp. 285 - 319.

[15] D. Norman, The psychology of everyday things. New York, NY, USA: Basic Books, 1988.

[16] J. Gibson, "The Theory of Affordances," in Perceiving, acting, and knowing: toward an ecological psychology, R. Shaw and University of Minnesota., Eds. Hillsdale, N.J., USA: Lawrence Erlbaum Associates, 1977.

[17] W. A. S. Buxton, "Integrating the Periphery and Context: A New Model of Telematics," in Proceedings of Graphics Interface - GI '95, 1995, pp. 239-246.

[18] M. Weiser, "Some Computer Science Issues in Ubiquitous Computing," Communications of the ACM, vol. 36, no. 7, pp. 7584, 1993.

[19] M. Langheinrich, "Privacy in Ubiquitous Computing," in Ubiquitous Computing Fundamentals, J. Krumm, Ed. Boca Raton, Florida, USA: CRC Press, 2010, pp. 37-94.

[20] J. R. Aiello, "Human Spatial Behaviour," in Handbook of environmental psychology, D. Stokols and I. Altman, Eds. New York: John Wiley \& Sons, 1987, pp. 359-504.

[21] H. Osmond, "Function as the basis of psychiatric ward design," Mental Hospitals (Architectural Supplement), vol. 8, pp. 23-29, 1957.

[22] E. T. Hall, "Proxemics," Current Anthropology, vol. 9, no. 2/3, pp. 83-108, Jun. 1968

[23] R. Sommer, Personal space: the behavioral basis of design. Englewood Cliffs, N.J: Prentice-Hall, 1969.

[24] T. M. Ciolek, "The proxemics lexicon: A first approximation," Journal of Nonverbal Behavior, vol. 8, no. 1, pp. 55-79, 1983.

[25] E. Hall, Handbook for Proxemic Research. Washington, DC, USA: Society for the Anthropology of Visual Communication, 1973.

[26] M. Baldassare, "Human Spatial Behavior," Annual Review of Sociology, vol. 4, no. 1, pp. 29-56, Aug. 1978.

[27] M. Argyle and J. Dean, "Eye-Contact, Distance and Affiliation," Sociometry, vol. 28, no. 3, pp. 289-304, Sep. 1965.

[28] E. Sundstrom and I. Altman, "Interpersonal relationships and personal space: Research review and theoretical model," Human Ecology, vol. 4, no. 1, pp. 47-67, Jan. 1976.

[29] I. Altman, The Environment and Social Behavior: Privacy, Personal Space, Territory, and Crowding. Brooks/Cole Publishing Company, Monterey, California, 1975.

[30] R. Want, A. Hopper, V. Falcao, and J. Gibbons, "The Active Badge Location System," ACM Transactions on Information Systems, vol. 10, no. 1, pp. 91-102, 1992.

[31] J. Müller, F. Alt, A. Schmidt, and D. Michelis, "Requirements and Design Space for Interactive Public Displays," in Proceedings of ACM Multimedia - MM '10, New York, NY, USA, 2010.

[32] T. Prante et al., "Hello. Wall-Beyond Ambient Displays," in Video and Adjunct Proceedings of Ubicomp Conference, 2003.

[33] H. Gellersen et al., "Supporting device discovery and spontaneous interaction with spatial references," Personal Ubiquitous Comput., vol. 13, no. 4, pp. 255-264, 2009.
[34] R. Vertegaal and J. S. Shell, "Attentive user interfaces: the surveillance and sousveillance of gaze-aware objects," Social Science Information, vol. 47, no. 3, pp. 275-298, Sep. 2008.

[35] N. Marquardt, R. Diaz-Marino, S. Boring, and S. Greenberg, "The Proximity Toolkit: Prototyping Proxemic Interactions in Ubiquitous Computing Ecologies," in Proceedings of UIST '11, ACM, 2011.

[36] C. Swindells, K. M. Inkpen, J. C. Dill, and M. Tory, "That one there! Pointing to establish device identity," in Proceedings of UIST '02, ACM, 2002, pp. 151-160.

[37] J. Rekimoto, Y. Ayatsuka, M. Kohno, and H. Oba, "Proximal Interactions: A Direct Manipulation Technique for Wireless Networking," in Proceedings of the IFIP International Conference on Human-Computer Interaction - INTERACT '03, IFIP, 2003, pp. 511-518.

[38] K. Hinckley, "Synchronous gestures for multiple persons and computers," in Proceedings of UIST '03, ACM, 2003, pp. 149158.

[39] L. Holmquist, F. Mattern, B. Schiele, P. Alahuhta, M. Beigl, and H.-W. Gellersen, "Smart-Its Friends: A Technique for Users to Easily Establish Connections between Smart Artefacts," in Proceedings of Ubicomp '01, 2001, p. 116.

[40] G. Ramos, K. Hinckley, A. Wilson, and R. Sarin, "Synchronous Gestures in Multi-Display Environments," Human-Computer Interaction, vol. 24, no. 1, p. 117, 2009.

[41] C. Kray, M. Rohs, J. Hook, and S. Kratz, "Group Coordination and Negotiation through Spatial Proximity Regions around Mobile Devices on Augmented Tabletops," in Proceedings of Tabletop '08, IEEE, 2008, pp. 1-8.

[42] M. Back, J. Cohen, R. Gold, S. Harrison, and S. Minneman, "Listen reader: an electronically augmented paper-based book," in Proceedings of CHI '01, ACM, 2001, pp. 23-29.

[43] C. Harrison and A. K. Dey, "Lean and zoom: proximity-aware user interface and content magnification," in Proceeding of $\mathrm{CHI}$ '08, ACM, 2008, pp. 507-510.

[44] H. A. He, "One Size Does Not Fit All: Extending the Transtheoretical Model to Energy Feedback Technology Design," MSc Thesis, Department of Computer Science, University of Calgary, 2010.

[45] E. Bardram, "The trouble with login: on usability and computer security in ubiquitous computing," Personal Ubiquitous Comput., vol. 9, no. 6, pp. 357-367, 2005.

[46] Y. Chen and M. Sinclair, "Tangible security for mobile devices," in Proceedings of the 5th Annual International Conference on Mobile and Ubiquitous Systems: Computing, Networking, and Services, Dublin, Ireland, 2008, pp. 1-4.

[47] R. Mayrhofer, H. Gellersen, and M. Hazas, "Security by Spatial Reference: Using Relative Positioning to Authenticate Devices for Spontaneous Interaction," in Proceedings of Ubicomp 2007, Springer, 2007, pp. 199-216.

[48] K. P. Fishkin, S. Roy, and B. Jiang, "Some Methods for Privacy in RFID Communication," in Security in Ad-hoc and Sensor Networks, 2005, pp. 42-53.

[49] N. Marquardt, A. Taylor, N. Villar, and S. Greenberg, "Rethinking RFID: Awareness and Control for Interaction with RFID Systems," in Proceedings of CHI '10, ACM, 2010, pp. 23072316. 